\title{
Physiological Changes in Bipolar Disorder
}

\author{
Marie Alexandra Vilbrun, RN, MSN*
}

PhD Student, Barry University, USA

*Corresponding author: Marie Alexandra Vilbrun, RN, MSN, PhD Student, Barry University, USA

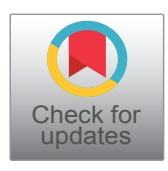

According to the World Health Organization, "an estimated 45 million people worldwide suffer from mental health disorders" [1]. Mental health disorders encompass the concept of mood and affect. Bipolar disorder is one exemplar of the mood and affect concept. According to the WHO [1], bipolar disorder consists of both manic and depressive episodes occurring between periods of a healthy mood. During manic episodes, patients may have elevated or irritable mood, hyperactivity, fast speech, delusions of grandeur, and decrease sleepiness. Some people who do have manic attacks but do not have depression are also diagnosed as suffering from bipolar disorder. Women have a higher incidence of bipolar disorder than men WHO [1]. The purpose of this integrative literature review is to understand the concept of mood and affect as it relates to bipolar disorder in women. This author reviews articles that depict various physiological changes that occur in women with bipolar disorder. Women of all walks of life have bipolar disorder, and nurses are often ill-prepared to deal with the complexities of their disorders. The psychiatric database APA PsycNET compiled many professional journals and yielded many peer-reviewed studies on the topic of bipolar disorder in women. The research articles were found while using the words "bipolar disorder "and "women" in the database search box. Despite a large number of studies identified, the author of this integrated literature review shall only use ten of them. This paper also talks about the relationship between women with bipolar disorder and sexual risks.

\section{Identification of the Concept}

\section{Bipolar disease}

Bipolar disorder is a severe illness that encompasses the concept of mood and affect. Researchers such as Minuzzi, et al. [2], Moreira, et al. [3], and Ceylan, et al. [4] agree that bipolar disorder is a debilitating chronic mental illness that is characterized by changes in physiology. Furthermore, they concur that the disease does not have any specific treatment approach. Treatment may need to be adjusted due to the stage of the disease process and other characteristics of patients. There is no one size fits all way to regulate this disorder. Mood and affect have to do with how individuals feel when faced with particular situations or independently of any external influencers. Changes in mood and affect are classic symptoms of this disease. According to the Encyclopaedia Britannica [5], during a depressive phase, sadness, listlessness, and lack of energy might be prominent. During a manic phase, elation, expansiveness, and irritability might manifest.

Regardless of the circumstances, some people have more vulnerable dispositions than others. The various feeling and affect imbalances can manifest themselves with rage, fits of hysteria, cries, and more. Physiological processes cause all these external signs of affective instability. Various triggers cause moods to fluctuate from one end of the spectrum to the other. It is challenging to regulate mood and affect as they involve complicated interactions between neurons, neurotransmitters, and various parts of the brain [6]. There is a pattern identified in the literature; it reveals how anatomical changes do contribute to the incidence of mood and affect issues, including bipolar disorder.

\section{Theoretical Perspectives and Patterns}

\section{Mood and affect}

The concept of mood and affect manifests in bipolar disorder. Many studies posit that bipolar disorder could be linked to the disruption of processes in the brain or other areas of the body. No specific theoretical

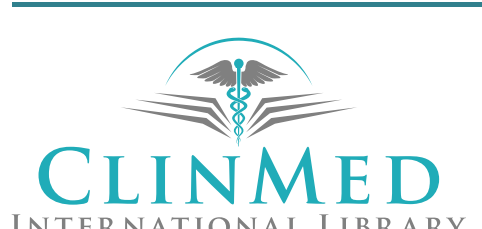

Citation: Vilbrun MA (2021) Physiological Changes in Bipolar Disorder. Int Arch Nurs Health Care 7:164. doi.org/10.23937/2469-5823/1510164

Accepted: August 19, 2021: Published: August 21, 2021

Copyright: (C) 2021 Vilbrun MA. This is an open-access article distributed under the terms of the Creative Commons Attribution License, which permits unrestricted use, distribution, and reproduction in any medium, provided the original author and source are credited. 
perspective was identified in the research studies. However, the perspective of psychiatry approach is an alternative approach to the DSM for the understanding and treatment of patients with psychiatric conditions [7]. It borrowed ideas from Dr. Meyer and Jaspers, famous psychiatrists of the 1900s could have been used as a theoretical framework for the research studies. The perspective of psychiatry approach considers disease process, pathological responses, patients' behavior, and background to guide studies on mood and affect [7]. That approach is a beneficial tool that is widely used by healthcare providers for the treatment and teaching of patients with psychiatric disorders. Patients with bipolar disorder often experience physiologic changes.

Lavagnino, et al. [8] mentioned that women in the late stage of bipolar disorder have more significant changes in the volume of their corpus callosum. Similarly, Minuzzi, et al. [2] describe the decrease in gray matter in the left somatosensory cortex of women with bipolar disorder. Ceylan, et al. [4] reported that women with bipolar disorder had accelerated oxidation induced DNA damage under the influence of stressors. Comparably, Moreira, et al., [3] described how nerve growth factor increases by $15.4 \%$ in women who converted from having a major depressive disorder to bipolar disorder. Patterns of physiological changes are identified in the studies mentioned above and will be discussed further in the research approaches part of this paper. Quantitative or qualitative methods were used and led to the conclusion that physiological changes occur with bipolar disorder. Other aspects of women life are affected by the presence of bipolar disorder.

\section{Relationship between Mood and Affect Concept and Other Phenomena}

\section{Lifestyle}

There is a positive relationship between women with bipolar disorder and an increase in sexual risks. Women, who suffer from mood disturbances, in this case, bipolar disorder, are more prone to careless sexual behavior according to the literature [9]. In their study, Marengo, et al. [10] compared two groups of sixty-three women. They used sixty-three female outpatients with bipolar disorder diagnosis from the bipolar program of Favaloro University. The other sixtythree women did not have any psychiatric history and led a regular healthy lifestyle. The sixty-three bipolar women practiced casual sex and were not in committed monogamous relationships. They also had intimate contacts with partners of undetermined HIV or other sexually transmitted diseases status. Furthermore, they were afflicted by two or more sexually transmitted infections. The authors concluded that women with bipolar disorder were more likely to participate in risky and promiscuous behavior compared to healthy women (women who do not have bipolar disorder).
Dinc, et al. [9] posit that women with bipolar disorder have poor preventive health habits as well as knowledge deficit. Dinc, et al. [9] compared 358 women with bipolar disorder with 346 healthy women. They determined that the bipolar cohort did not know much about the date around which they started menstruating. They also did not have regular periods and seldom went to get preventive screenings such as mammograms and other gynecological tests. Similarly to the Marengo, et al. research, this study also found that the bipolar group of women had more sexually transmitted infections and more sexual mates than their healthy counterparts. Bipolar disorder causes women to have poor judgment, behave with impulsivity, and deal with hypersexuality and illicit substances [11]. This study also concluded that bipolar women had more debilitating symptoms before menstruations and tended to masturbate more than the healthy cohort of women. Other reproductive risks are associated with bipolar disorder.

The risk of unintended pregnancy is higher among women with bipolar disorder for at least two reasons. Their permissive sexual behaviors could cause these women to get pregnant at any time during their reproductive years. Furthermore, Miller, et al. [11] wrote that there are significant drug interactions between mood stabilizers and contraceptives. Estrogen based contraceptive potentiate the effect of some mood stabilizers. Mood stabilizers can cause neural tube defects, hepatotoxicity, and other congenital disabilities in bipolar women fetuses. These facts contribute to the lack of compliance with medicines by women who have bipolar disorder.

Another study mentioned that there is a correlation between hysterectomy and the incidence of bipolar disorder occurring after the procedure. The research conducted by Shen, et al. [12] described this phenomenon also related to the concept of mood disorder/bipolar. The researchers compared 4,337 women (aged 30 to 50) who had a hysterectomy with 17,348 women who did not have a hysterectomy. They concluded that women who received a hysterectomy were more at risk of developing bipolar disorder. They also mentioned that endometriosis and hormone replacement therapy might contribute to the highest incidence of mood disorder post-hysterectomy.

The study by Miller, et al. [11] concluded that postpartum and bipolar episodes could occur concurrently. They also said that the risk of relapse is higher during post-partum for women who have bipolar disorder. Symptoms can manifest as early as four weeks postdelivery [13]. The same study also asserted that women with bipolar disorder are more prone to psychosis. Symptoms of psychosis include hallucinations, delusions, mood lability, impaired judgment, and more. A plethora of dangerous mood manifestations can quickly put a newborn at risk of harm or death. Other phenomena are 
also associated with the presence of bipolar disorder. Miller, et al. [11] posit that women with bipolar disorder have a higher incidence of obesity, which can increase their risk of dying from cardiovascular diseases.

\section{Four Research Studies that Support Alterations in Mood and Affect Concept}

\section{Women and bipolar disorder}

Ceylan, et al. [4] presented research on increased plasma levels of 8-oxoguanine DNA glycosylase-1 in bipolar disorder. Forty-eight women with bipolar disorder and forty-nine healthy women were included in this project. Excluded from the study were women with other psychiatric issues, infections, neurological disorders, history of head trauma or brain surgery, chronic medical disorder, substance abuse disorder, ongoing pregnancy, or were breastfeeding. Each woman provided a $10 \mathrm{ml}$ blood sample, which was analyzed for 8-OHdG and OGG1 protein (prevalent protein evidence for DNA base damage).

The women with bipolar and healthy women were similar as far as age, sex, and body mass were concerned. There were a higher number of smokers in the women with the bipolar group vs. the healthy women group. The plasma level of OGG1 protein was higher in the women with the bipolar group. The presence of increased OGG1 protein in women with bipolar disorder suggests that they have a higher risk for DNA oxidation/damage compared to healthy women. This study did not mention any limitations. The authors cited a need for further research involving brain tissue. They also suggested the use of other methods to assess DNA damage susceptibility.

Lavagnino, et al. [8] conducted a study on the changes in the corpus callosum of women with latestage bipolar disorder. A convenient sample of adult women aged 19 to 65 was divided into three groups. One group included 20 women with early-stage bipolar disease, a second group consisted of 21 women with late-stage bipolar, and a healthy group of 25 women made the third group. Women with other neurological histories, head trauma, major medical illnesses, or were unable to have magnetic resonance imaging (MRI) were excluded.

Corpus callosum represents the most significant white matter part of the brain and plays a role in language, attention, memory, emotion, and many more brain functions. This area of the brain is very susceptible to stress and trauma. This study and others suggested that the size of the corpus callosum of women with late bipolar disorder was smaller in volume. This finding was based on MRI data comparing the brains of the three groups of women included in the study. This study limitation included a cross-sectional design, the inability to study specific aspects of the corpus callosum, the lack of data on traumatic events in the life of the participants, and the lack of specificity between early and late bipolar determination. A longitudinal study could bring better information on the progress of bipolar disease upon women's brains, particularly women's corpus callosum.

Moreira, et al. [3] researched the serum level of nerve growth factor as a biomarker in the conversion of women from major depressive disorder to bipolar disorder. This longitudinal study included 156 women aged 18 to 60 years and had three years follow up. The sample was chosen by convenience, and the experiment done in two phases. All participants were invited to both stages, but eight had to be excluded for a couple of reasons. Questionnaires were used to collect various demographics, and trained psychologists administered a mini neuropsychiatric test. The women were divided into three groups remitted major depressive disorder (MDD), current MDD, and diagnostic conversion to bipolar disorder (BD).

Various blood samples were collected via an immune essay kit. They measured serum interleukin-6, tumor necrosis factor-alpha, brain-derived neurotrophic factor, glial cell neurotrophic factor, and nerve growth factor. The conversion rate from major depressive disorder (MDD) to bipolar disorder (BD) was $15 \%$, and the level of nerve growth factor was higher in these women. The limitations of this study included: A convenience sample/small sample size, limited family history of bipolar disorder, limited information on the age of onset of depressive episodes. Other limitations involve limited knowledge about women's thyroid function, peripartum, and sex hormones.

Minuzzi, et al. [2] explored structural and functional changes in the somatosensory cortex of euthymic women with bipolar disorder. A total of 68 euthymic (tranquil mental state) women were included in this study. Bipolar disorder was diagnosed in 32 of these women, and 36 women were healthy. The study was done to evaluate the volume of gray matter (GM) and resting-state functional connectivity (Rs-FC) in the cohorts. The hypothesis was that women with bipolar disorder would show a decrease in GM and abnormal Rs-FC at the somatosensory cortex and limbic regions compared to other healthy women of the same age.

All participants signed informed consent before participating in the research study. The exclusion criteria for bipolar women included current mood issues (depression, hypomania, mania). These exclusions also included psychiatric comorbidities, changes in psychotropic medications, unstable medical conditions. Exclusion criteria for the healthy women included the presence of any mental issue during their life and any unstable medical conditions. The exclusions for all women comprised current or recent use of hormonal treatment, pregnancy, and inability to get magnetic resonance imaging (MRI). They also included any history of head trauma involving loss of consciousness, 
neurological disorder impairing cognition, and alcohol or drug abuse or dependence.

Psychiatric, menstrual cycle phase and depressive symptoms were all assessed using appropriate measuring tools. MRI data were collected via a GE high-performance scanner. All images and eye-open functional resting-state were taken via a high-resolution process. All tests conducted in this study were advanced and highly technical. After the study, researchers suggested that women with bipolar disorder had higher RS-FC in the regions (right somatosensory cortex \& front limbic) responsible for affective regulation. However, the same women had decreased gray matter (GM) in the left somatosensory cortex compared to the healthy group.

The limitation of this study includes a cross-sectional design, the limited sample size, inability to assess medication influence, and a strictly female cohort. Future research should explore the emotional regulation task and the inclusion of male participants. These new variables would bring more findings and knowledge for the treatment and management of bipolar disorder.

\section{Summary and Recommendations}

Millions of women worldwide suffer from major depressive disorders, including bipolar disorder. Many studies discussed the effects of disruptive physiological changes as being the trigger or precursor of this debilitating mood disorder. Bipolar disorder is also linked to a vast array of other phenomena which can affect women's life. The research studies reviewed above implied that women with bipolar disorder are a cohort at risk for severe complications, including morbidities and mortality. These negatives prospects include increased risk of sexually transmitted infections, unintended pregnancies, and deficient preventive health habits. Additionally, the likelihood of side-effects from medications, and relapse after delivery or hysterectomy can be added to the plethora of issues leading to the diagnosis of bipolar disorder. The researches assessed in this literature review were mainly focused on the physiologic changes occurring with women with bipolar disorder. The studies were reliable and valid; the conclusions were logical and consistent with the findings. A pattern was identified as all the studies revealed a correlation between physiological alterations and the presence of bipolar disorder in women.

For the purpose of this literature review, only studies focusing on physiology were reviewed. Any additional phenomenon associated with this disease, such as highrisk sexual behavior, lifestyle changes, and more would contribute to a holistic picture of determining best practices in caring for women with bipolar. Significant milestones have been accomplished in the area of brain analysis and physiological changes regarding bipolar disorder. However, there is much more that as yet to be discovered about bipolar disorder. More research is needed to address the effect of ethnicity, culture, and religion on mood and affect in bipolar disorder. Any new research within these parameters will add new knowledge that could benefit treatments and management of the disorder in various cohorts.

\section{References}

1. World Health Organization (2020) Mental disorders.

2. Minuzzi L, Syan SK, Smith M, Hall A, Hall GBC, et al. (2018) Structural and functional changes in the somatosensory cortex in euthymic females with bipolar disorder. Aust N Z J Psychiatry 52: 1075-1083.

3. Moreira FP, Cardoso TC, Mondin TC, Wiener CD, de Mattos Souza LD, et al. (2019) Serum level of nerve growth factor is a potential biomarker of conversion to bipolar disorder in women with major depressive disorder. Psychiatry Clin Neurosci 73: 590-593.

4. Ceylan D, Yilmaz S, Bora U, Tuna G, Ildiz A, et al. (2019) Increased plasma levels of 8-oxoguanine DNA glycosylase-1 in bipolar disorder. Psychiatry Clin Neurosci 73: 719-720.

5. (2020) Bipolar disorder. In: Encyclopaedia Britannica online.

6. Giddens JF (2017) Concepts for nursing practice. Elsevier, St. Louis, MI.

7. Peters ME, Taylor J, Lyketsos CG, Chisolm MS (2012) Beyond the DSM: The perspectives of psychiatry approach to patients. Prim Care Companion CNS Disord 14: 01233.

8. Lavagnino L, Cao B, Mwangi B, Wu MJ, Sanches M, et al. (2015) Changes in the corpus callosum in women with latestage bipolar disorder. Acta Psychiatr Scand 131: 458-464.

9. Dinc H, Boyacioglu NE, Ozcan NK, Enginkaya S (2019) Reproductive and sexual health in women with bipolar disorder: A comparative study. Dusunen Adam the Journal of Psychiatry and Neurological Sciences 32: 23-32.

10. Marengo E, Martino DJ, Igoa A, Fassi G, Scapola M, et al. (2015) Sexual risk behaviors among women with bipolar disorder. Psychiatry Res 230: 835-838.

11. Miller LJ, Ghadiali NY, Larusso EM, Wahlen KJ, AvniBarron O (2015) Bipolar disorder in women. Health Care for Women International 36: 475-498.

12. Shen YC, Chen W, Tsai IJ, Wang JH, Lin SZ, et al. (2019) Association of hysterectomy with bipolar disorder risk: A population-based cohort study. Depress Anxiety 36: 543551.

13. Di Florio A, Forty L, Gordon-Smith K, Heron J, Jones L, et al. (2013) Perinatal episodes across the mood disorder spectrum. JAMA Psychiatry 70: 168-175. 